FULL TEXT ARTICLE

\title{
Adherence to risk evaluation and mitigation strategies (REMS) requirements for monthly testing of liver function
}

\author{
Christopher M Blanchette, ${ }^{1}$ Anthony P Nunes, ${ }^{2}$ Nancy D Lin, ${ }^{2}$ Kathleen M Mortimer, ${ }^{2}$ Joshua Noone, ${ }^{1}$ \\ Krishna Tangirala, ${ }^{3}$ Stephen Johnston, ${ }^{4}$ Benjamin Gutierrez ${ }^{3}$ \\ ${ }^{1}$ University of North Carolina, Charlotte, NC, USA; ${ }^{2}$ Optum Epidemiology, Waltham, MA, USA; \\ ${ }^{3}$ Otsuka America Pharmaceutical, Inc., Princeton, NJ, USA; ${ }^{4}$ Truven Health Analytics, Washington, DC, USA
}

\section{Citation}

Blanchette CM, Nunes AP, Lin ND, Mortimer KM, Noone J, Tangirala K, Johnston S, Gutierrez B. Adherence to risk evaluation and mitigation strategies (REMS) requirements for monthly testing of liver function. Drugs in Context 2015; 4: 212272 . doi: 10.7573/dic. 212272

\section{Copyright}

Copyright ( 2015 Blanchette CM, Nunes AP, Lin ND, Mortimer KM, Noone J, Tangirala K, Johnston S, Gutierrez B. Distributed under the terms of the Creative Commons License Deed CC BY NC ND 3.0 which allows anyone to copy, distribute, and transmit the article provided it is properly attributed in the manner specified below. No other uses without permission.

\section{Correct attribution}

Copyright @ 2015 Blanchette CM, Nunes AP, Lin ND, Mortimer KM, Noone J, Tangirala K, Johnston S, Gutierrez B. http://dx.doi.org/10.7573/dic.212272. Published by Drugs in Context under Creative Commons Attributions License Deed CC BY NC ND 3.0.

\section{Article URL}

http://www.drugsincontext.com/adherence-risk-evaluationmitigation-strategies-rems-requirements-monthlytesting-liver-function

\section{Correspondence}

Christopher M Blanchette, Associate Dean for Research \& Public Engagement, Director of Data Sciences \& Business Analytics, Associate Professor of Public Health Sciences, College of Health \& Human Services, University of North Carolina at Charlotte, 9201 University City Boulevard, CHHS 476, Charlotte, NC 28223, USA. cblanche@uncc.edu

\section{Provenance}

Submitted, externally peer reviewed

\section{Dates}

Submitted: 2 December 2014

Accepted, subject to peer review: 8 December 2014

Revised manuscript submitted: 29 December 2014

Publication date: 10 February 2015

\section{Publisher \& contact information}

Drugs in Context is published by Just Medical Media Ltd, Undermount, Rydal, Ambleside, Cumbria, LA22 9LT, UK; ISSN 1740-4398; Just Medical Media Limited is registered in England Number 6891187; VAT GB 945171322

\section{Julia Savory}

Head of Digital Publishing and Submissions Management julia@justmedicalmedia.com; Tel: +44 (0)1242910999

\section{Abbreviations}

ALT, alanine aminotransferase; AST, aspartate aminotransferase; ETASU, elements to ensure safe use; FDA, Food and Drug Administration; IQR, interquartile range; LFT, liver function test; ORD, Optum Research Database; $\mathrm{PAH}$, pulmonary arterial hypertension; REMS, risk evaluation and mitigation strategies; SD, standard deviation; TAP, Tracleer (bosentan) Access Program 


\section{Group Editor-in-Chief}

\section{Christopher Blanchette, PhD, MBA}

Associate Dean for Research and Research Associate Professor in the Department of Public Health Sciences at the University of North Carolina and Director of Health Economics \& Outcomes Research at Otsuka America Pharmaceutical Inc, USA

\section{Expert Advisers - Epidemiology and biostatistics}

\section{Alex K Exuzides, PhD}

Director, ICON Clinical Research Inc, California, USA

\section{Professor Scott L Friedman, MD}

Fishberg Professor of Medicine, Dean for Therapeutic Discovery Chief, Division of Liver Diseases, Mount Sinai School of Medicine, New York, USA

\section{Carl De Moor, PhD}

Senior Principal, Epidemiology and Leader Epidemiology, Safety and Risk Management Center of Excellence Americas, IMS Health Inc, USA

\section{Dr John H Walker, OCT, MBA, PhD}

Professor, Goodman School of Business, Brock University,

St Catharines, Ontario, Canada

\section{Specialist editorial board members}

\section{Deborah P Lubeck, PhD}

Vice President, Outcomes Insights Inc, CA, USA

\section{Specialist Advisor - Clinical Pharmacology}

Dr Richard White, MA, PhD

Consulting Partner and Director, Oxford PharmaGenesis Ltd, UK

To see the full Drugs in Context Editorial Board, please visit www.drugsincontext.com/editorial-board

\section{Expert Adviser - Publication Ethics}

\section{Dr Elizabeth (Liz) Wager}

Publications Consultant, Princes Risborough, UK; Visiting Professor, University of Split School of Medicine, Croatia; Former Chair (2009-2012), Committee on Publication Ethics (COPE)

\section{Editor-in-Chief Emeritus \\ Dr George Kassianos, FRCGP, FBHS, FESC, FBGTHA, FAcadMEd, FFTM RCPSGlasg}

General Practitioner, Bracknell, Berkshire, UK; President, British Global \& Travel Health Association; Fellow of the European Society of Cardiology 


\title{
Adherence to risk evaluation and mitigation strategies (REMS) requirements for monthly testing of liver function
}

\author{
Christopher M Blanchette, ${ }^{1}$ Anthony P Nunes, ${ }^{2}$ Nancy D Lin, ${ }^{2}$ Kathleen M Mortimer, ${ }^{2}$ Joshua Noone, ${ }^{1}$ \\ Krishna Tangirala, ${ }^{3}$ Stephen Johnston, ${ }^{4}$ Benjamin Gutierrez ${ }^{3}$ \\ ${ }^{1}$ University of North Carolina, Charlotte, NC, USA; ${ }^{2}$ Optum Epidemiology, Waltham, MA, USA; \\ ${ }^{3}$ Otsuka America Pharmaceutical, Inc., Princeton, NJ, USA; ${ }^{4}$ Truven Health Analytics, Washington, DC, USA
}

\section{Citation}

Blanchette CM, Nunes AP, Lin ND, Mortimer KM, Noone J, Tangirala K, Johnston S, Gutierrez B. Adherence to risk evaluation and mitigation strategies (REMS) requirements for monthly testing of liver function. Drugs in Context 2015; 4: 212272 . doi: $10.7573 /$ dic. 212272

\section{Abstract}

Background: Risk evaluation and mitigation strategies (REMS), as mandated by the US Food and Drug Administration (FDA) for medications with the potential for harm, are increasingly incorporating rigid protocols for patient evaluation, but little is known about compliance with these programs. Despite the inherent limitations, data on administrative claims may provide an opportunity to investigate adherence to these programs.

Methods: We assessed adherence to liver function test (LFT) requirements included in the REMS program for bosentan through use of administrative claims. Patients observed in the Optum Research Database who were initiators of bosentan from November 20, 2001 to March 31, 2013 were included. Adherence to LFTs was calculated using pharmacy claims for bosentan dispensation and medical claims for laboratory services, and was assessed the time of drug initiation and within specified time intervals throughout follow-up.

Results: Of 742 patients, 523 (70.5\%) had $\geq 1$ qualifying LFT. Among patients with $\geq 12$ dispensations, claims for LFTs at individual dispensations were $53.2-64.0 \%$. Median proportion of dispensations with $\geq 1$ LFT was 0.8 among patients with $\geq 6$ (interquartile range, $0.7-1.0$ ) or $\geq 12(0.7-0.9)$ dispensations. Adherence was $90-100 \%$ for $33.3 \%$ of all initiators, whereas $29.3 \%$ of initiators were non-adherent (defined as $<50 \%$ of on-therapy LFTs).

Conclusions: Analyses of administrative claims suggest that the REMS program for bosentan may not have adequately guaranteed adherence to the program's monthly monitoring of LFTs. Such investigations of existing REMS programs may provide insight on how to accomplish more successful evaluation of REMS.

Keywords: risk evaluation, REMS, liver function test, adherence, compliance, FDA, patient assessment, administrative claims

\section{Introduction}

An important function of the US Food and Drug Administration (FDA) is to manage and minimize the risk for potential adverse events from approved drugs. FDA Amendments Act of 2007 gave the FDA the authority to mandate incorporation of risk evaluation and mitigation strategies (REMS) for drugs with the potential for harm [1]. All REMS programs must have a "minimal strategy" timetable for assessment at defined intervals, and can also include one or more of the following: medication guide; communication plan; elements to ensure safe use (ETASU); an implementation plan for ETASU $[2,3]$. The various elements of REMS programs are designed to ensure safe use of a product and can include: restricted access; healthcare provider or pharmacy certifications; regular monitoring of patients [2,3].
The REMS process is evolving, and the lack of standardization often complicates implementation of individual programs [3]. Safest possible use of treatments can be achieved through collaboration of all parties: patient, provider, manufacturer, and the FDA. Recently, the FDA has required REMS programs to include rigid protocols for patient evaluation as a condition for continued dispensation of the covered medication. Protocols may include required laboratory tests and, if pre-specified thresholds are met, termination of the medication is required. These protocols can be valuable in reducing risk to patients but only if they are targeted successfully toward, and adhered to by, the particular patient population at risk for specific adverse events, along with the treating physician. To ensure that the precautions put in place by REMS are being implemented appropriately, adherence to their requirements should be 
monitored and, if necessary, actions should be taken to improve deficiencies. Little is known about adherence to REMS programs by patients and providers [4]. The present study was designed to evaluate the level of adherence to specific requirements within a rigid REMS program.

Bosentan is an endothelin receptor antagonist indicated for treatment of pulmonary arterial hypertension (PAH) [5]. Bosentan has one of the most restrictive REMS programs of any medication on the market, including multiple requirements regarding potential risks for hepatotoxicity and birth defects [6]. Bosentan is available only through the Tracleer (bosentan) Access Program (TAP), which requires provider attestation of liver function testing before initiation of bosentan use and patient attestation of monthly assessments during therapy. One element of REMS and the TAP requires providers to have reviewed pretreatment liver function tests (LFTs) and ordered monthly LFTs for anticipated duration of therapy ( $\leq 1$ year) before first prescribing bosentan to a patient. Despite these requirements for bosentan, a "Dear Doctor" letter was issued in 2006 to report labelling changes based on rare cases of hepatotoxicity as well as to remind providers about the importance and frequency of required LFTs [7]. A more stringent modification of REMS was issued in 2012, which added elements to improve adherence with LFT assessments [6].

The bosentan REMS program was evaluated in the present study because its rigid protocols for patient assessment and medication access may serve as a model for future program development. In an effort to assess adherence to LFT within this REMS program and changes in adherence after communications and actions regarding regulation of drug safety, we used data based on administrative claims (a common data source for pharmacoepidemiologic studies and evaluation of drug safety).

\section{Methods}

\section{Study design}

This observational study used data regarding eligibility, pharmacy, and medical claims from the Optum Research Database (ORD), representing patients from a large US health plan. The ORD is a proprietary database with geographically diverse enrollment data from 1993 to the present day. Data relating to $\approx 12.6$ million individuals with medical and pharmacy benefit coverage are available for 2012.

Study population comprised a cohort of patients who received at least one dispensation of bosentan between November 20, 2001 and March 31, 2013 (Figure 1). Patients must have had $\geq 90$ days of medical coverage and pharmacy benefits before cohort entry. Patients also had to be aged $<65$ years and have no additional public or private insurance. Bosentan dispensations were identified within pharmacy claims through Hierarchical Ingredient Codes Lists. Initiators of bosentan were defined as those with $\geq 1$ pharmacy claim for dispensation of bosentan and no prior dispensation within 90 days. The index date was the date of the first eligible dispensation within the study period. Patients were followed up until the earliest of the following: discontinuation of bosentan therapy, disenrollment, or the end of the study period (March 31, 2013). Discontinuation of bosentan was assumed if the gap between refills exceeded the days supplied plus a 10-day grace period. Therefore, the period of observation for each individual was restricted to the first interval of continuous bosentan therapy.

LFT characterization included laboratory claims for aspartate aminotransferase (AST) and alanine aminotransferase (ALT), as well as for panels that included AST or ALT. Assessments attempted to replicate the monitoring requirements set forth in the bosentan REMS program in how the measure was defined, but the timing was varied conservatively [6]. Laboratory tests for levels of AST and ALT were identified on the basis of Current Procedural Terminology codes for individual tests and for laboratory panels present in outpatient medical claims.

Figure 1. Study methodology.

\section{Bosentan dispensations}

- Identified within pharmacy claims November 20, 2001 through March 31, 2013

- $\geq 1$ dispensation and no prior dispensation within 90 days

- Index date of the first eligible dispensation within the study

- Final analysis dispensations were those within continuous therapy

$\downarrow$

\section{ALT or AST laboratory tests}

- Identified based on CPT codes for individual tests and for laboratory panels within outpatient medical claims

- Service dates for LFTs obtained from 90 days prior to initiation through the earliest of bosentan discontinuation, termination of pharmacy or medical coverage, or March 31, 2013

- Inpatient stays of 2 days or more were assumed to include an LFT

Assessments
- Intervals were anchored at each dispensation and
defined as the shorter of the predetermined interval
length or the time since previous dispensation
- Separate analyses were performed using a 40-day
or 35-day interval
- Patient-level adherence to REMS LFT requirements
was calculated as the proportion of dispensations with
a qualifying prior LFT (number of dispensations with
a prior LFT divided by total number of dispensations)

ALT, alanine amino transferase; AST, aspartate aminotransferase; CPT, current procedural terminology; LFT, liver function test; REMS, risk evaluation and mitigation strategies; TAP, Tracleer (bosentan) Access Program. 
Laboratory panels containing ALT and/or AST are commonly ordered during inpatient stays. However, data on inpatient claims do not reliably contain information on specific laboratory tests due to the bundling of service charges. Therefore, inpatient stays of $\geq 2$ days were assumed to include a LFT.

To assess adherence, patient follow-up was divided into mutually exclusive intervals anchored the time of each bosentan dispensation. Interval lengths were defined as the shorter of either 40 days before dispensation or the time since the previous dispensation. Sensitivity analyses were undertaken using a 35-day interval. LFT adherence associated with the index dispensation of bosentan was assessed over a 90-day interval. Adherence to LFT requirements within REMS was calculated using pharmacy claims for bosentan dispensation and medical claims for laboratory services. Binary indicators of $\geq 1$ LFT were generated for the index dispensation and each subsequent refill. Within patients, adherence to guidelines for LFT monitoring was calculated as the number of bosentan dispensations with an associated prior LFT divided by the total number of bosentan dispensations.

\section{Analyses}

Distributions of LFT adherence were analyzed overall and observed by time periods of interest. Analyses were conducted using SAS* 9.2. Distributions were compared, but no formal tests were conducted to evaluate determinants of adherence by statistical means. Reported measures included proportions, means, and standard deviations (SDs), as well as medians and interquartile ranges (IQRs). Characteristics of the study population that were summarized included: demographics; type of provider of index dispensation; medical utilization; medical diagnoses during baseline and follow-up.

Adherence classifications were defined as "high" (90-100\%), "moderate" (75-89\%), "low" (50-74\%), and "non-adherent" $(<50 \%)$. Adherence per year was evaluated by year of index dispensation (adherence per patient was categorized into the year of the patient's date of initiation). Regulatory events of interest during the study period included: a Dear Doctor letter issued in March 2006; expansion of indications in August 2009 to include milder forms of PAH; REMS modification in October 2012 to add elements to improve compliance with LFT assessments.

\section{Results}

\section{Characteristics of the study and Patients}

The study population consisted of 742 patients in whom bosentan therapy had been initiated (Table 1). Consistent with the indications for bosentan, most patients had a diagnosis of chronic pulmonary heart disease or PAH. Most prescribing

*SAS Institute Inc., Cary, NC, USA.
Table 1. Demographic characteristics of bosentan initiators within the Optum Research Database from November 20, 2001 to March 31, 2013.

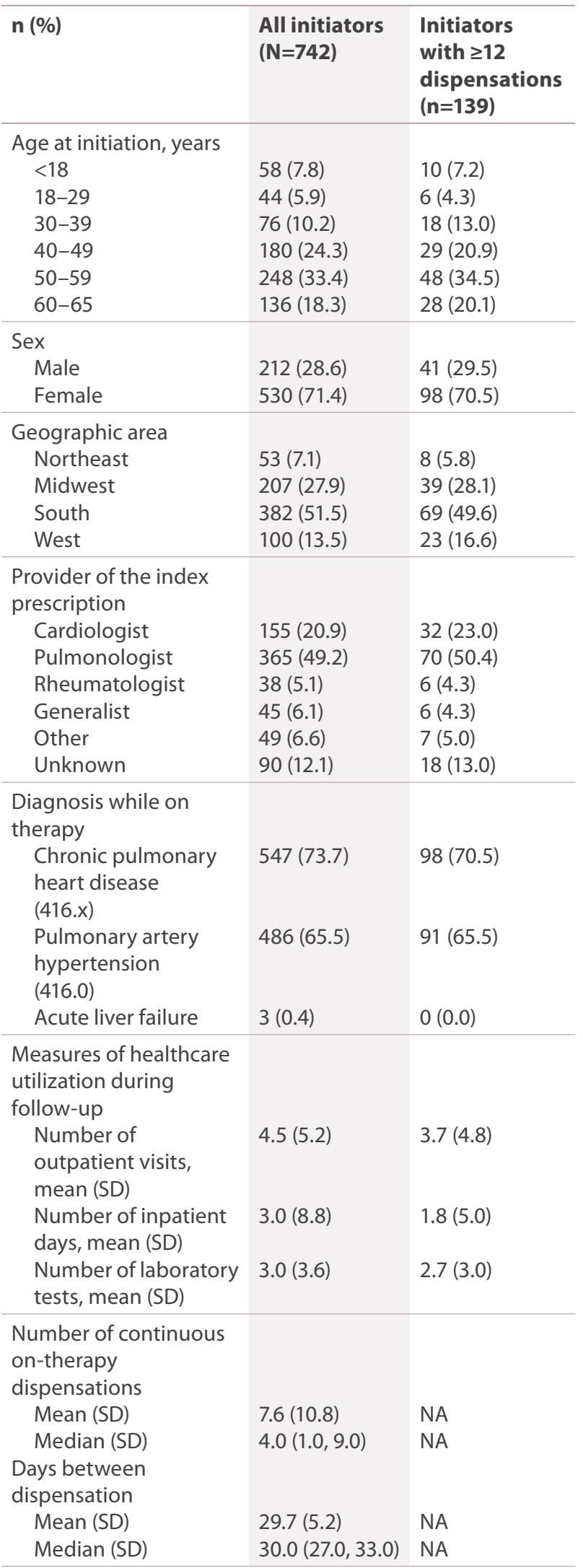

NA, not available; SD, standard deviation. 
Table 2. Characterization of bosentan dispensations and relative timing of liver function tests within the Optum Research Database from November 20, 2001 to March 31, 2013.

\begin{tabular}{|c|c|c|c|c|}
\hline & \multirow{2}{*}{$\begin{array}{l}\text { Patients with } \\
\text { dispensation } \\
\text { (n) }\end{array}$} & \multirow{2}{*}{$\begin{array}{l}\text { Patients with a LFT within } \\
\text { the previous } 40 \text { days } \\
\text { (n) }\end{array}$} & \multicolumn{2}{|c|}{$\begin{array}{c}\text { Days between dispensation and most } \\
\text { proximal prior LFT }\end{array}$} \\
\hline & & & Mean (SD) & Median (p25, p75) \\
\hline $\begin{array}{l}\text { Index } \\
\text { dispensation* }\end{array}$ & 742 & 523 & $26.1(22.9)$ & $18.0(8.0,40.0)$ \\
\hline 1st refill & 538 & 285 & $10.0(8.3)$ & $8.0(3.0,16.0)$ \\
\hline 2nd refill & 469 & 265 & $12.4(8.9)$ & $11.0(5.0,19.0)$ \\
\hline 3rd refill & 394 & 237 & $12.8(9.2)$ & $12.0(4.0,20.0)$ \\
\hline 4th refill & 334 & 193 & $12.6(9.2)$ & $12.0(4.0,20.0)$ \\
\hline 5th refill & 290 & 182 & $13.6(8.9)$ & $13.0(6.0,20.0)$ \\
\hline 6th refill & 251 & 150 & $13.2(9.3)$ & $12.0(5.0,20.0)$ \\
\hline 7th refill & 226 & 139 & $13.4(9.2)$ & $13.0(6.0,21.0)$ \\
\hline 8th refill & 209 & 117 & $12.3(9.3)$ & $11.0(5.0,20.0)$ \\
\hline 9th refill & 184 & 106 & $13.0(9.0)$ & $13.0(6.0,20.0)$ \\
\hline 10th refill & 147 & 87 & $12.7(8.8)$ & $12.0(6.0,20.0)$ \\
\hline 11th refill & 139 & 85 & $11.8(9.3)$ & $9.0(4.0,18.0)$ \\
\hline 12th refill & 129 & 73 & $12.6(9.4)$ & $10.0(4.0,21.0)$ \\
\hline
\end{tabular}

*Within a 90-day interval for the index dispensation.

LFT, liver function test; SD, standard deviation.

providers were cardiologists or pulmonologists. Patients were admitted for a mean of 3 inpatient days, which is consistent with the severity of the primary indication for bosentan. Median number of bosentan dispensations was 4 (IQR, 1.0-9.0) and the distribution time between consecutive dispensations centered on 30 days. Median time between bosentan dispensation and a prior LFT was longer for the index dispensation compared with the median time between dispensation and a prior LFT for the first 12 refills (Table 2). However, assessment of the index dispensation was defined as a 90-day interval compared with the 40-day interval for refill dispensations. Mean number of days between dispensation and the most proximal prior LFT was 10.0-13.6.

\section{Adherence to LFTs}

Of the 742 patients, 523 (70.5\%) had at $\geq 1$ qualifying LFT before the index dispensation (Table 3). In the 40-day assessment, among patients with $\geq 12$ dispensations, the percentage of patients with a claim for a LFT was lowest at dispensation number 8 (53.2\%) and highest at dispensation number 3 (64.0\%). Adherence (defined for each person as the median proportion of dispensations with $\geq 1$ LFT) was 0.8 among patients with $\geq 6$ (IQR 0.7-1.0) or $\geq 12$ (0.7-0.9) dispensations. Among all initiators, $33.3 \%$ had high adherence $(90-100 \%$ of dispensations had a corresponding LFT), whereas $29.3 \%$ of all initiators were considered to be non-adherent $(<50 \%$ of bosentan dispensations had a corresponding LFT). The level of adherence was similar whether the assessment used 40or 35-day intervals.

Aside from years 2001 and 2013 (for which patient numbers were low), annual classification of adherence was: $26.8-41.9 \%$ for patients classified as highly adherent; $6.8-25.0 \%$ for those classified as moderately adherent; $14.5-32.3 \%$ for those classified as low adherent; $16.7-39.0 \%$ for those classified as non-adherent (Figure 2). Among patients considered to be highly adherent, adherence was highest among those who initiated in the years immediately after the initial approval for bosentan, with another peak for those initiating in 2010, for which $41.9 \%$ patients were considered to be highly adherent. Changes in LFT adherence were not observed after identified events of interest, including a Dear Doctor letter reminding providers about the importance and frequency of required LFTs (March 2006) and an expansion of indications to include milder forms of PAH (August 2009).

\section{Discussion}

In general, REMS initiatives are imperative to ensuring patient safety. Investigation of individual REMS programs can lead to better future programs with the potential to increase patient safety, and permit use of medications that may confer benefit in select populations despite inherent risk. Adherence by physicians and patients to specified requirements is critical to the effectiveness of a REMS program. Hence, we investigated adherence to a restrictive REMS program implemented for 
Table 3. Assessment of adherence* to monitoring of liver function within the Optum Research Database from November 20, 2001 to March 31, 2013.

\begin{tabular}{|c|c|c|}
\hline & $\begin{array}{l}\text { All initiators } \\
(\mathrm{N}=742)\end{array}$ & $\begin{array}{l}\text { Initiators with } \geq 12 \text { dispensations } \\
(n=139)\end{array}$ \\
\hline \multicolumn{3}{|c|}{$\geq 1$ LFT within dispensation-specific assessment intervals, n (\%) } \\
\hline Index dispensation & $523(70.5)$ & $99(71.2)$ \\
\hline 1st refill & $285(53.0)$ & $77(55.4)$ \\
\hline 2nd refill & $265(56.5)$ & $84(60.4)$ \\
\hline 3rd refill & $237(60.2)$ & $89(64.0)$ \\
\hline 4th refill & $193(57.8)$ & $83(59.7)$ \\
\hline 5th refill & $182(62.8)$ & $81(58.3)$ \\
\hline 6th refill & $150(59.8)$ & $79(56.8)$ \\
\hline 7th refill & 139 (61.5) & $81(58.3)$ \\
\hline 8th refill & $117(56.0)$ & $74(53.2)$ \\
\hline 9th refill & $106(57.6)$ & 79 (56.8) \\
\hline 10th refill & $87(59.2)$ & $80(57.6)$ \\
\hline 11th refill & 85 (61.2) & 85 (61.2) \\
\hline 12th refill & 73 (56.6) & $73(56.6)$ \\
\hline
\end{tabular}

\section{Proportion of dispensations with $\geq 1$ prior LFT while continuously on therapy}

\begin{tabular}{lll}
\hline $\begin{array}{l}\text { All dispensations } \\
\text { Mean (SD) } \\
\text { Median (p25, p75) }\end{array}$ & $0.6(0.4)$ & $0.6(0.3)$ \\
$\begin{array}{c}\text { First } 6 \text { dispensations } \\
\text { Mean (SD) }\end{array}$ & $0.7(0.3,1.0)$ & $0.6(0.3,0.8)$ \\
$\quad \begin{array}{l}\text { Median (p25, p75) } \\
\text { First 12 dispensations }{ }^{\ddagger}\end{array}$ & $0.8(0.2)$ & $0.7(0.2)$ \\
$\quad$ Mean (SD) & $0.8(0.7,1.0)$ & $0.7(0.7,0.8)$ \\
$\quad$ Median (p25, p75) & $0.8(0.2)$ & $0.8(0.2)$ \\
\hline
\end{tabular}

\section{Categories of LFT adherence}

\begin{tabular}{llr}
\hline High: $90-100 \%$ of on-therapy LFTs & $247(33.3)$ & $15(10.8)$ \\
Moderate: $75-89 \%$ of on-therapy LFTs & $105(14.2)$ & $35(24.2)$ \\
Low: $50-74 \%$ of on-therapy LFTs & $173(23.3)$ & $41(29.5)$ \\
Non-adherent: $<50 \%$ of on-therapy LFTs & $217(29.3)$ & $48(34.5)$
\end{tabular}

*Based on 40-day intervals except for the index dispensation, which was a 90 -day interval. ${ }^{\dagger}$ Restricted to patients with $\geq 6$ dispensations of bosentan. ${ }^{\ddagger}$ Restricted to patients with $\geq 12$ dispensations of bosentan.

LFT, liver function test; SD, standard deviation.

bosentan. The REMS program for bosentan is a model program in terms of complexity and requirements. However, when based on evaluation of data of administrative claims, these findings suggest that providers and patients may not fully adhere to these requirements.

An early study following the mandate to authorize the FDA to implement REMS revealed significant concerns among stakeholders from various perspectives - healthcare providers, drug sponsors, patient advocates, payers, and pharmacists that REMS would have significant impacts on the healthcare system [2]. Current studies highlight the steps being proposed and taken to improve REMS programs, particularly to standardize programs and evaluate effectiveness [3,4]. The FDA recently introduced a REMS Integration Initiative to evaluate and improve implementation of REMS programs [8].
Several studies have also begun to analyze various aspects of the FDA REMS program. Secular trends in REMS approval, assessment of approved REMS characteristics, and time lags between drug approval and REMS approval were investigated through data collected from FDA Approved Drug Products with Therapeutic Equivalence Evaluations [9]. That particular study reported FDA-approved REMS for 1 in 3 biologics and 1 in 13 chemical entities available in the US market, and that the number of pharmaceuticals with serious risk requiring REMS increased significantly over time. However, recent policy changes by the FDA with regard to some medication guides not requiring REMS indicate that a full REMS will be reserved for a small number of high-risk medications [10]. The International Society for Pharmacoeconomics and Outcomes Research Risk Benefit Management Working Group analyzed and compared the FDA's REMS program and the European Medicines Agency's 
Figure 2. Temporal changes in adherence by calendar year* of the index date and by adherence periods of LFTs as defined by FDA inquiries and action ${ }^{\dagger}$ within the Optum Research Database from November 20, 2001 to March 31, 2013.

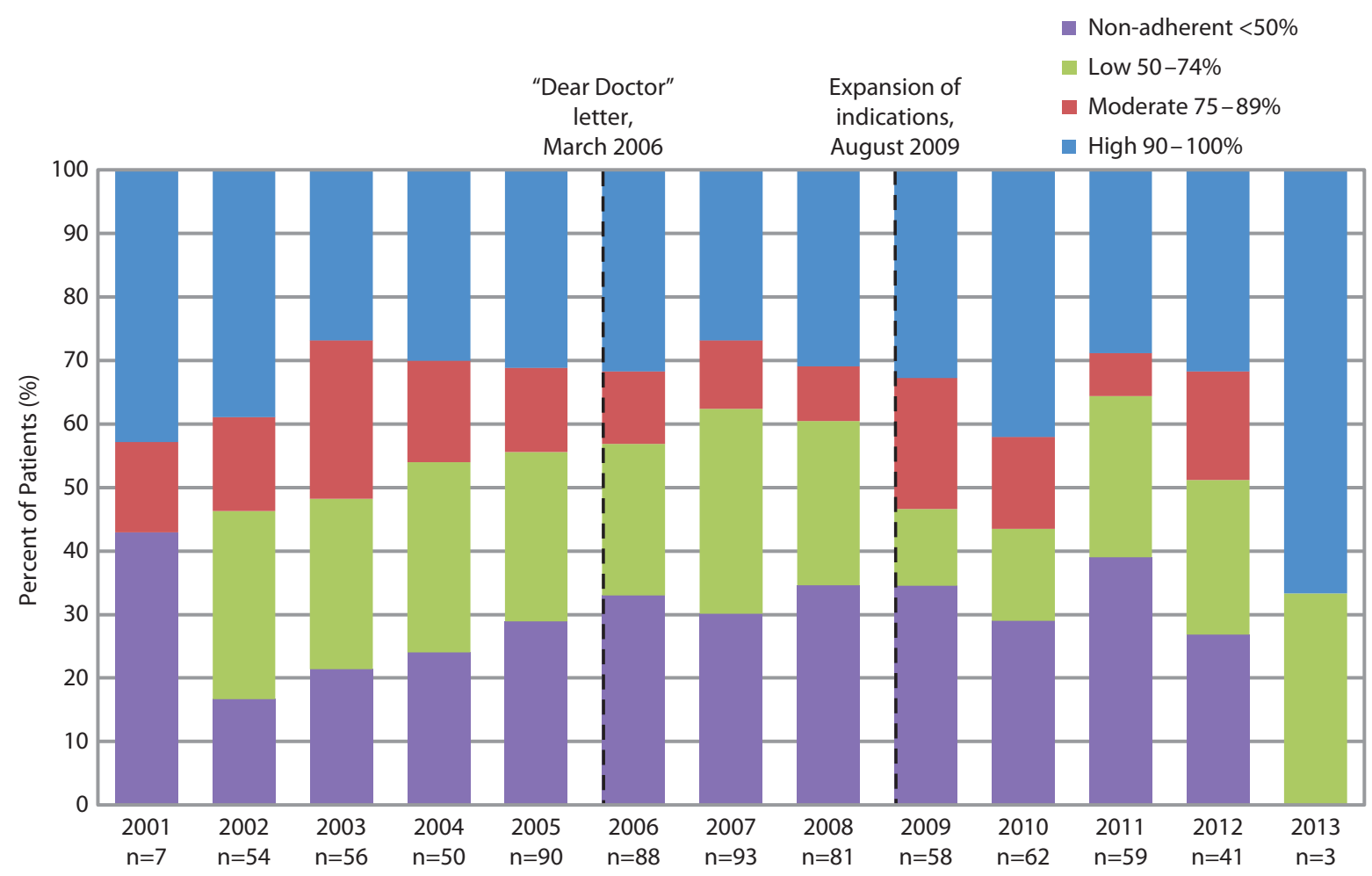

*Based on 40-day intervals; adherence per patient was categorized into the year of the patient's date of initiation. ${ }^{\dagger}$ Regulatory events of interest during the study period include a Dear Doctor letter issued in March 2006 and expansion of indications to include milder forms of PAH in August 2009.

FDA, Food and Drug Administration; LFT, liver function test; REMS, risk evaluation mitigation strategies.

Risk Management Plan [11]. They found that both provide positive guidance for identifying, monitoring, and minimizing risks to patient safety, but neither provides specific guidance on how risk should be balanced with benefit, either qualitatively or quantitatively [11]. Risk communication is an integral part of REMS programs, but the educational effectiveness of the approach has not been studied extensively. A recent investigation analyzed the approach of adding an Internetbased continuing-medical-education activity, and found improvement in the understanding of specific drug toxicities among healthcare providers [12].

In the present study, adherence to an example of a stringent program that requires LFT monitoring was investigated utilizing only data on administrative claims. Adherence to REMS requirements for LFT monitoring while on continuous therapy was $70.5 \%$. In the context of the present study, the evidence did not suggest meaningful improvement in adherence following periods defined by FDA inquiries and actions pertaining to LFTs. As sensitivity analyses, data from another database on administrative claims representing a different patient population were assessed. The Truven Health Marketscan Commercial administrative claims database comprises administrative claims from $\approx 40$ million employees and dependents covered annually under various health insurance plans. Among 660 patients with a bosentan dispensation between October 1, 2009 and September 30, 2012, adherence to LFTs within 35 days before dispensation was $50.3 \%$ in patients with $\geq 1$ dispensation (data not shown). Adherence increased to $62 \%$ if considering inpatient stays as unobserved (but potentially valid) LFT events. For patients who underwent all 12-treatment cycles $(n=228), 61.4 \%$ were adherent; this value increased to $64.9 \%$ with incorporation of tests during hospitalization. Adherence levels observed in these sensitivity analyses were slightly lower than those observed using the ORD.

It is clear that an ongoing tracking system can provide more accountability and reconciliation of adherence with REMS programs due to the inability of readily available data sources to completely capture non-adherence by patients and physicians. Studies to evaluate these systems are becoming more prevalent. For example, a study covering 2005-2011 for evaluation of asthma medications found that the implemented REMS program resulted in a reduction of use of fluticasone propionate/salmeterol and 
encouraged appropriate use of long-acting $\mathrm{B}_{2}$-adrenergic agonists, a major goal of the REMS program [13]. Even with manufacturers and the FDA working together to develop REMS programs to ensure patient safety, managing the risk effectively without full engagement of the patient and provider (as well as a method to track such an engagement) is difficult.

Investigation of REMS programs (and especially adherence to their measures) is necessary. Databases of administrative claims (such as those used in our study) are a convenient and cost-effective method of investigation. However, interpretation of findings based solely on electronic claims should consider the inherent limitations in the processing and accumulation of these data. Claims for LFTs were observed, but claims data do not enable assessment of whether LFTs were reviewed by the provider, or whether the provider responded appropriately to elevated laboratory results, which is required by the REMS and which would provide more complete analyses of adherence. In addition, claims-based data include medication dispensations, but there is no assurance that the medication was taken as prescribed. Furthermore, the ORD includes a large and diverse patient population, but patients within this analytic dataset may not fully represent the patterns of adherence observed in the general population.

A LFT claim is strongly indicative that an LFT occurred, but additional variability may be present due to unobserved testing. In the present study, data for patients aged $>65$ years were excluded, and results were limited to the subset that was commercially insured without dual coverage from other insurers (government, commercial, private). This restriction was intended to limit the possibility of observing a claim for a dispensation of bosentan within the ORD among patients for whom laboratory testing is covered by an alternative insurer (and therefore not observed as a claim within the ORD). Consistent with this hypothesis, sensitivity analyses involving patients with co-insurance yielded notably lower estimates of adherence. The restriction of dual insurance limited the possibility of unobserved fulfillment of testing requirements, but the possibility remains that patients could have received tests in another manner (e.g., via manufacturer-sponsored programs and free clinics), which is a limitation of use of claims data.

Investigating existing REMS programs with rigid protocols may provide insight on how to accomplish more successful implementation of REMS with similar restrictions. Taking into account the limitations of using claims data, the results of this study indicate less-than-optimal adherence. Joint efforts between the FDA and pharmaceutical companies (including efforts to increase adherence from patients and providers) may make improvement of REMS more successful.

\section{Contributions}

Drs. Blanchette, Gutierrez, Mortimer, and Nunes contributed to the conception and design of this study. Contributions to acquisition of data were made by Dr. Nunes. Contributions to statistical analyses were made by Drs. Mortimer and Nunes. Supervisory contributions were made by Drs. Blanchette, Lin, Mortimer, Noone, and Nunes. Contributions related to administrative, technical or material support were made by Dr. Johnston. All authors contributed to the writing, review, and revision of the manuscript. Drs Blanchette, Gutierrez, Johnston, Lin, Mortimer, Nunes, and Tangirala contributed to analyses and interpretation of data.

\section{Potential conflicts of interest}

The International Committee of Medical Journal Editors' (ICMJE) Potential Conflicts of Interests forms for the authors are available for download at: http://www.drugsincontext.com/ wp-content/uploads/2015/02/dic.212272-COI.pdf

Christopher M Blanchette and Benjamin Gutierrez are former employees of Otsuka America Pharmaceutical, Inc. Anthony Nunes, Kathleen Mortimer, and Nancy Lin are employees of Optum Epidemiology and Stephen Johnston is an employee of Truven Health Analytics; both companies have received funds from Otsuka America Pharmaceutical, Inc. in connection with the conduct of this study. Joshua Noone received funds from Otsuka America Pharmaceutical, Inc. in connection with the conduct of this study. Krishna Tangirala is an employee of Otsuka America Pharmaceutical, Inc.

\section{Funding declaration}

This study was sponsored by Otsuka America Pharmaceutical, Inc. (Princeton, NJ, USA). Medical writing and editorial support for preparation of this manuscript was provided by Scientific Connexions, Inc. (Lyndhurst, NJ, USA), funded by Otsuka America Pharmaceutical, Inc. None of the material in this manuscript has been presented previously.

\section{Acknowledgements}

The authors acknowledge Scientific Connexions, Inc. for assistance in the preparation of this manuscript.

\section{References}

1. U.S. Department of Health and Human Services Food and Drug Administration. PUBLIC LAW 110-85-SEPT. 27, 2007. Washington, DC; September 2007. Available at: http://www.gpo.gov/fdsys/pkg/PLAW-110publ85/pdf/ PLAW-110publ85.pdf [Last accessed: October 22, 2014].

2. Wilson A, Milne CP. FDA's risk evaluation and mitigation strategies (REMS): effective and efficient safety tools or process poltergeist? Food Drug Law J 2011;66(4):569-85.

3. Nelson LS, Loh M, Perrone J. Assuring safety of inherently unsafe medications: the FDA risk evaluation and mitigation strategies. J Med Toxicol 2014;10(2):165-72. http://dx.doi.org/10.1007/s13181-013-0374-z 
4. Banerjee AK, Zomerdijk IM, Wooder S, Ingate S, Mayall SJ. Post-approval evaluation of effectiveness of risk minimization: methods, challenges and interpretation. Drug Saf 2014;37(1):33-42. http://dx.doi.org/10.1007/s40264-013-0126-7

5. Actelion Pharmaceuticals US, Inc. TRACLEER (package insert). South San Francisco, CA: 2012.

6. Actelion Clinical Research Inc. New supplement for NDA 21-290 Tracleer (bosentan), Risk Evaluation and Mitigation Strategy. Cherry Hill, NJ: 2012.

7. Segal ES. Dear healthcare professional letter regarding revisions to the Tracleer (bosentan) prescribing information concerning importance of continued monthly liver function testing. South San Francisco, CA: Actelion Pharmaceuticals US; 2006.

8. U.S. Department of Health and Human Services Food and Drug Administration. REMS Integration Initiative. Washington, DC; September 2014. Available at: http://www.fda.gov/ForIndustry/UserFees/ PrescriptionDrugUserFee/ucm350852.htm [Last accessed: October 22, 2014].

9. Rodriguez-Monguio R, Spielberger K, Seoane-Vazquez E. Examination of risk evaluation and mitigation strategies and drug safety in the US. Res Social Adm Pharm 2014;10(1):232-8. http://dx.doi.org/10.1016/j.sapharm.2013.03.005
10. U.S. Department of Health and Human Services Food and Drug Administration. Guidance Medication Guides. Washington, DC; November 2011. Available at: http://www.fda.gov/downloads/Drugs/ GuidanceComplianceRegulatorylnformation/ Guidances/UCM244570.pdf [Last accessed: October 22, 2014].

11. Lis Y, Roberts MH, Kamble S, Guo J, Raisch DW. Comparisons of Food and Drug Administration and European Medicines Agency risk management implementation for recent pharmaceutical approvals: report of the International Society for Pharmacoeconomics and outcomes research risk benefit management working group. Value Health 2012;15(8):1108-18. http://dx.doi.org/10.1016/j.jval.2012.06.019

12. Kraus CN, Baldwin AT, McAllister RG Jr. Improving the effect of FDA-mandated drug safety alerts with internetbased continuing medical education. Curr Drug Saf 2013;8(1):11-16.

http://dx.doi.org/10.2174/1574886311308010003

13. DiSantostefano RL, Yeakey AM, Raphiou I, Stempel DA. An evaluation of asthma medication utilization for risk evaluation and mitigation strategies (REMS) in the United States: 2005-2011. J Asthma 2013;50(7):776-82. http://dx.doi.org/10.3109/02770903.2013.803116 